\title{
Inaccuracies in Facial Recognition of Fear and Sadness for Male Domestic Violence Offenders
}

\author{
Blair Nyline, Lia Softas-Nall, Eric Peterson, Mackenzie Peake, Christy Jersin Woods \\ Department Applied Psychology and Counselor Education, University of Northern Colorado, Greeley, CO, USA \\ Email: basilia.softas-nall@unco.edu
}

How to cite this paper: Nyline, B., Softas-Nall, L., Peterson, E., Peake, M. and Woods, C.J. (2018) Inaccuracies in Facial Recognition of Fear and Sadness for Male Domestic Violence Offenders. Open Journal of Social Sciences, 6, 37-51.

https://doi.org/10.4236/jss.2018.62004

Received: December 6, 2017

Accepted: January 30, 2018

Published: February 2, 2018

Copyright () 2018 by authors and Scientific Research Publishing Inc. This work is licensed under the Creative Commons Attribution International License (CC BY 4.0).

http://creativecommons.org/licenses/by/4.0/

\section{Open Access}

\begin{abstract}
The purpose of this study was to compare males who were court ordered into domestic violence treatment (domestic violence offenders; DVOs) due to family violence against their female partner or spouse $(n=35)$ and a group of nonviolent males $(n=35)$ on facial emotion recognition and measures of self-identified empathy, cognitive ability, trauma history, and demographic information. A significant difference was found between the two groups in that DVOs were significantly less accurate in identifying sadness and fear, and identifying emotions of female faces compared to male faces. DVOs were also less accurate in identifying emotions at $40 \%$ and $60 \%$ emotional intensity when six primary emotions were combined. Clinical implications of the study include emphasizing emotion recognition in treatment for DVOs in order to ameliorate family violence.
\end{abstract}

\section{Keywords}

Domestic Violence, Emotion Recognition, Self-Identified Empathy, Cognitive Ability

\section{Introduction}

Domestic violence has been a predominant emotional, systemic, and financially costly problem in the United States. While at its basic form, domestic violence is about partners harming one another, it is also viewed as a problem of men's oppression of women, a broad public health problem, a criminal justice problem, and an economic problem both for the victims and society (Whitaker \& Lutzker, 2009) [1]. In the United States, $24.3 \%$ of women have experienced severe physical aggression by a partner, and $48.4 \%$ have experienced psychological aggression by a partner (Jaffe, Simonet, Tett, Swopes, \& Davis, 2015) [2]. Moreover, some individuals convicted of domestic violence may have a significant history 
of physical and emotional abuse as children (Gardner, Moore \& Dettore, 2014) [3]. Previous research on facial emotion recognition found that abused and neglected children were less accurate in recognizing facial emotions (Luke \& Banerjee, 2013) [4].

There is a great deal of literature on empathy in violent criminal offenders, specifically sex offenders and those diagnosed with antisocial personality disorder (Barnett \& Mann, 2013 [5]; Gery, Miljkovitch, Berthoz, \& Soussignan, 2009 [6]). Research involving domestic violence offenders (DVOs) suggests that empathy, or the lack of empathy, may play a role in one's offense (Covell, Huss, \& Langhinrichsen-Rohling, 2007) [7], yet little research has been done to explore the relationship between empathy and family violence. Understanding the role of empathy in domestic offenses is important for preventative work, as well as treatment of DVOs to ameliorate family violence. The study of empathy in DVOs can be used to highlight the potential that empathy may have in overpowering the desire to offend. Previous research with violent offenders has found a correlation between a low level of emotional intelligence and aggressive behavior (Jaffe et al., 2015) [2]. Accurately identifying the emotions of others is the first step in an effective empathic response which may later has a preventative impact on family violence.

Written measures and facial emotion measures are often compared to assess similarities or differences in perceived empathic ability and actual emotion recognition. The findings have produced mixed results. Some studies have found that offenders rate lower in both written empathy measures and facial affect recognition tasks than nonviolent controls, while other studies have found that offenders score similarly to nonviolent controls in both written empathy measures and facial emotion identification tasks (Book et al., 2007 [8]; Brook \& Kosson, 2012 [9]; Marsh \& Blair, 2008 [10]). It is important to clarify these conflicting findings to ascertain the hypothesis that empathy plays a significant role in violent offending. In order to understand how, and to what extent, empathy may play a role in family violence, it is first necessary to understand how accurately DVOs recognize facial emotions.

\subsection{Present Study}

In the present study, domestically violent male offenders were compared to nonviolent male controls on ability to accurately identify facial emotions in relation to their self-identified level of empathy. Ability to accurately identify the emotions of others was also compared after controlling for cognitive ability and demographic factors such as: trauma history, age, race and ethnicity, income, education, and chemical dependence history. Based on previous research of domestic offenders, we hypothesized that DVOs would be less accurate in identifying the emotions of others compared to nonviolent men, even after controlling for cognitive and demographic differences (Book et al., 2007 [8]; Brook \& Kosson, 2012 [9]; Marsh \& Blair, 2008 [10]). It was also hypothesized that there 
would be no significant differences in self-identified level of empathy.

\subsection{Empathy and Recognizing Facial Emotions}

Current models of treatment approach DVO therapy from a cognitive behavioral model, incorporating psychoeducation about violence and power differentials between the genders (Bowen, Gilchrist, \& Beech, 2005 [11]; Gardner et al., 2014 [3]). There is little focus on understanding the victim's emotions or victim empathy. Moreover, treatment focuses minimally on interpersonal reaction and understanding one's partner (Bowen et al., 2005) [11]. A literature review on empathy in DVOs found a lack of literature on empathy and domestic violence. Specifically, the relationship between self-reported empathy for DVOs and his/her ability to recognize the emotions of others has not been explored in current literature.

Facial emotion recognition tasks have been used with other types of violent offenders to increase their knowledge of how they interact with others and help gain understanding around why they may become violent. Facial emotion recognition tasks are a measure of cognitive empathy, defined as the ability to identify how another person may be feeling (Barnett \& Mann, 2013 [5]; Domes, Hollerbach, Vohs, Mokros, \& Habermeyer, 2013 [12]). Examining a DVOs ability to accurately identify facial emotion and comparing this to his/her perceived empathic ability is an important step forward in improving our understanding of family violence etiology.

To date, a thorough literature review has resulted in only one study examining the ability of DVOs to accurately identify facial emotions (Babcock, Green, \& Webb, 2008) [13]. Participants in the study were assessed on their ability to accurately identify facial emotions compared to other men with varying degrees of violent behavior. The researchers found that domestically violent men categorized as "generally violent" struggled to accurately identify angry, happy, neutral, and surprised emotional expressions (Babcock et al., 2008) [13]. Domestically violent men who were categorized as "only violent in the family setting" or had predominant "borderline personality characteristics" were not found to have deficits in accurately identifying facial emotions.

\section{Method}

\subsection{Participants}

Participants were a sample of 35 adult DVOs from the Rocky Mountain region in court mandated treatment following a domestic violence conviction. In addition, they were classified as Level C offenders, which means that they were at the highest risk of committing another domestic violence offense. Control participants were 35 complete samples of adult men recruited using a snowball sampling method. Exclusion criteria for the control sample were a history of perpetration of violence as indicated by self-report (Hooper, Stockton, Krupnick, \& Green, 2011) [14]. It was a requirement of all participants to have the ability to 
read and speak English fluently given that literacy is necessary to read and understand the Interpersonal Reactivity Index (Lauterbach \& Hosser, 2007) [15].

In this study, the age for DVO participants ranged from 22 to $62(\mathrm{M}=35.29$, $\mathrm{SD}=9.73$ ), while age for the nonviolent control participants ranged from 19 to $69(\mathrm{M}=37.37, \mathrm{SD}=15.7)$. Participants were mostly Caucasian, $51 \%$ and $77 \%$ for DVO and nonviolent men, respectively. For the remaining DVO participants, $37 \%$ identified as Hispanic or Latino, 9\% identified as multiracial, and 3\% identified as "other". For the remaining nonviolent control participants, $6 \%$ identified as Hispanic or Latino, 6\% identified as African American, 3\% identified as Asian, 3\% identified as Native American, 3\% identified as multiracial, and 3\% identified as "other". The majority (53\%) of the control participants' annual family income was greater than $\$ 85,000$ a year while most of the domestic violence offender's annual income was in the \$10,000 - 24,999 range (32\%) and the $\$ 25,000$ - 39,999 (32\%) range. Much of the domestic violence offender participants had some college education (37\%), while control participants had either some college education (43\%) or a college degree (57\%). Information on chemical dependence was gathered via self-report. Fifty-seven percent of the DVO participants reported a history of chemical dependence while only $7 \%$ of the control participants reported a history of chemical dependence.

\subsection{Procedure}

DVO participants were recruited through Domestic Violence Offender Management Board (DVOMB) approved treatment providers in the Rocky Mountain region. Directors of treatment agencies that provide domestic violence treatment were contacted directly and informed of the purpose of the study and of IRB approval. When granted approval, participants were recruited directly from individual treatment groups. In each group, mandated DVOs were informed of the purpose of the study and asked to participate. They were informed that their participation was completely voluntary and would not affect their treatment, parole, or probation. Control participants were recruited using a snowball sample from the lead researchers' professional contacts. Control and DVO participants were asked to schedule time for participation during recruitment, and asked to leave contact information for a reminder phone call. The identifying information was destroyed upon data collection.

Before data collection, participants were given the informed consent and had all questions answered. Participants were given a large battery of surveys including a demographic questionnaire, and the Life Events Checklist-5 (LEC-5), the Interpersonal Reactivity Index (IRI), and the General Ability Measure for Adults (GAMA) measures described below. Once participants finished the written measures, they were told they would be shown a series of photographs on a laptop computer depicting men and women displaying various emotional expressions. Each participant sat in front of a laptop computer approximately two feet from the screen. They were asked to maintain that distance and continue to 
sit straight to maintain the integrity of the measure. A picture depicting a specific emotion was presented one at a time to each participant. Using a forced-choice response format, participants were asked to assess each emotion in the photograph concurrent to its presentation by pressing the button indicating the correct emotion. Each emotion corresponded with a number on the laptop keyboard. Once each participant identified the emotion, the screen transitioned to the next face. Each face was presented in random order for each participant. Each participant completed a practice facial recognition task before the data collection began to record results. During the practice task, the lead researcher stood next to participants and requested they identify the emotion out loud to ensure the emotion they identified corresponded with the correct number on the keyboard. Once the practice task was completed, participants were no longer asked to identify the emotion out loud and the lead researcher sat next to the participant. Upon task completion, participants were debriefed regarding the purpose of the study, given their five-dollar incentive, and entered the twenty-five-dollar raffle if they chose to do so.

\subsection{Measures}

Demographics Questionnaire. All participants completed a brief demographic questionnaire developed by the researchers. Information covered in the demographic questionnaire included race and ethnicity, native language, annual family income, education, and a history of drug or alcohol abuse. Demographics were collected to analyze differences between the domestic violence offender participants and the nonviolent control participants and whether demographics would impact the accuracy of facial emotion recognition.

Life Events Checklist-5 (LEC-5). The Life Events Checklist-5 (LEC-5) is a measure designed to identify one's exposure to traumatic events over the course of his or her lifetime. It is a 17 -item measure that lists possibly traumatic events and asks the participant to identify if the event "happened to me", "witnessed it", "learned about it", "not sure", and "doesn't apply". Participants are asked to identify which category each event falls under over the course of his or her lifetime. The LEC-5 has strong correlational properties with other popular measures of Posttraumatic Stress Disorder (PTSD) symptoms (Gray, Litz, Hsu, \& Lombardo, 2004) [16].

Interpersonal Reactivity Index (IRI). The Interpersonal Reactivity Index (IRI) is a measure of perceived empathy commonly used in research with the offender population (Lauterbach \& Hosser, 2007) [15]. The IRI is a 28-question measure of empathy that includes four dimensions: empathic concern, perspective taking, personal distress, and fantasy (Davis, 1983) [17]. Empathic concern is defined as one's affective response to another person's emotional state or feelings of compassion or concern. Perspective taking is viewed as the cognitive skill of taking the viewpoints of others and comprehending their situation without the need of comprehending corresponding feelings. Personal distress is the ten- 
dency to experience distress and discomfort in response to stressful situations. Fantasy is the reaction to fictional instead of real situations such as movies, plays, and books. It is the tendency to involve oneself in the feelings and actions of fictitious characters (Davis, 1983). Internal reliabilities range from 0.71 to 0.77 and the test-retest reliabilities range from 0.62 to 0.71 .

General Ability Measure for Adults (GAMA). The General Ability Measure for Adults (GAMA) is a nonverbal assessment of general cognitive ability (Naglieri \& Bardos, 1997) [18]. It "evaluates an individual's overall general ability with items that require the application of reasoning and logic to solve problems that exclusively use abstract designs and shapes" (Bardos, 2003, p. 164) [19]. The assessment is comprised of 66 items categorized into four item types called Matching, Analogies, Sequences, and Construction (Bardos, 2003) [19]. According to Bardos (2003) [19], the assessment was designed to reduce the number of confounds that come with a language-based assessment. Language items in an assessment can be influenced by one's exposure to a formal English speaking academic environment regardless of his or her true cognitive ability. Another goal of the assessment was to reduce the influence of motor ability and speed at the item level by eliminating the use of manipulated objects. The average reliability across the item types was 0.65 for Construction, 0.66 for Matching, 0.79 for Sequences, and 0.81 for Analogies. In terms of validity, the GAMA scores have consistently been similar to the Wechsler Adult Intelligence Scales (WAIS-R) and (WAIS-III), as well as the Kaufman Adolescent and Adult Intelligence Scale (KAIT).

Emotion Recognition Items. Emotion recognition items were taken from the Nimstim data set (Tottenham et al., 2009) [20]. The Nimstim data set builds on previous facial expression data sets such as Ekman and Friesen (1978) [21], and addresses many of the criticisms of these earlier data sets. For example, the Nimstim data set is available in color and contains a large number of stimuli and variety of facial expressions. Validity and reliability for the Nimstim data set were calculated using a representative group of undergraduate college students from the Midwest and a volunteer sample from the East Coast. Validity was calculated by finding the percentage of correctly identified emotions while using Cohen's kappa to account for false positives by chance (Tottenham et al., 2009) [20]. The mean kappa percentage correct across stimuli was 0.79 . Reliability was calculated by presented stimuli in a different random order in its entirety after a 20 -minute break. This second presentation of stimuli was not used to calculate validity scores. The reliability score or proportion of agreement had a mean of 0.84 (Tottenham et al., 2009) [20].

The facial emotion recognition task included six primary emotions of interest: sadness, fear, disgust, anger, happiness and surprise. Neutral was not included in order to keep the assessment as brief as possible while maintaining adequate sensitivity in the task. Participants within the normal population are typically very accurate at identifying the six primary emotions in data sets in which the 
emotion is shown at $100.0 \%$ intensity. New research has begun to blend the $100.0 \%$ intensity emotions with a neutral face using face-morphing technology resulting in facial blends that demonstrate the emotion at a lower level of intensity. The result is a more accurate demonstration of emotion that is more likely to be seen in daily interactions (Gery et al., 2007 [6]; Pham \& Philippot, 2010 [22]).

The data set used in the current study consisted of a set of faces developed by Peterson and Peake (Peterson \& Peake, 2015) [23]. Peterson and Peake constructed the facial affect recognition task based on guidelines set by previous literature. Specifically, an equal number of male and female faces were presented (Babcock et al., 2008 [13]; Gery et al., 2009 [6]), the percentage of facial blends was set at 30.0\%, 40.0\%, 60.0\%, 70.0\%, and 100.0\% (Gery et al., 2009 [6]; Tottenham, et al., 2009 [20]), and the specific emotions included in the task were sadness, anger, surprise, fear, disgust, and happiness (Babcock et al., 2008 [13]; Marsh \& Blair, 2008 [10]).

Six Caucasian faces from the standardized Nimstim prototypic facial expressions were used. The faces were blended using six universal emotions (happiness, sadness, anger, disgust, fear, and surprise) to $30.0 \%, 40.0 \%, 60.0 \%, 70.0 \%$, and $100.0 \%$ intensity (Tottenham et al., 2009) [20]. Based on the neutral face $(0.0 \%$ of emotional intensity) and the full emotional facial expression $(100.0 \%$ emotional intensity) of the same actor, the computer program E-Prime (http://www.pstnet.com/eprime.cfm) was used to construct blends of each emotion at the increments mentioned above. The different intensity of emotions was modeled from Pham and Philippot (2010) [22] who indicated, “...Such full-blown displays have little ecological validity" because they are easy to decode and are likely to produce ceiling effects and leave little room for individual variance (p. 448). The images were also masked by Peterson and Peake with a dark oval around the face to control for the possible distraction of jewelry, hair, or other factors not directly related to the facial affect. Each emotion (anger, fear, disgust, surprise, sadness, and happiness) at each level of emotional intensity $(30.0 \%, 40.0 \%, 60.0 \%, 70.0 \%$, and $100.0 \%)$ were shown six times using an equal number of male and female models ( 3 male, 3 female) for a total of 180 images.

\section{Results}

A total of 70 men completed all aspects of data collection and were included in the study. Half of the participants $(n=35)$ were in court-mandated treatment due to family violence at the time of data collection. The control group $(n=35)$ was a sample of men who did not have a history of perpetration of violence as indicated by self-report. Multivariate analyses of variances (MANOVAs) were conducted comparing differences in facial emotion accuracy by emotion type and facial emotion intensity by violence status (domestically violent vs. nonviolent). Analyses of covariance (ANCOVA) were conducted comparing differences in emotion identification accuracy while controlling for cognitive and demo- 
graphic differences. Finally, a $2 \times 2$ analyses of variance (ANOVA) was conducted to identify any differences in emotion identification accuracy by facial target (male or female).

\subsection{Facial Emotion Identification}

A MANOVA used to identify specific differences in facial emotion recognition between DVO participants and control participants revealed that DVO participants were significantly less accurate in identifying the emotions of fear $(F(1,68)$ $=5.49, p=0.02)$ and sadness $(F(1,68)=5.47, p=0.02)$. There was no significant difference in facial emotion recognition for the emotions of anger $(F(1,68)=$ $0.52, p=0.47)$, disgust $(F(1,68)=1.36, p=0.25)$, surprise $(p=0.49)$, or happiness $(F(1,68)=2.00, p=0.16)$. Participants were most accurate in identifying happiness (DVO participants $M=0.83$; control participants $M=0.87$ ) and least accurate in identifying fear (DVO participants $M=0.38$; control participants $M$ $=0.48$ ). Figure 1 shows the comparison between groups on overall facial emotion recognition.

A MANOVA was used to identify differences in emotional recognition at varying degrees of emotional intensity. There was no significant difference in accuracy of emotion recognition at the highest $(100 \%, F(1,68)=1.05, p=0.309)$ and lowest $(30 \%, F(1,68)=2.87, p=0.095)$ levels of emotional intensity. There was also no significant difference between the groups at $70 \%$ emotional intensity $(F(1,68)=3.54, p=0.064)$. DVO participants were significantly less accurate than the nonviolent control group in identifying the emotions of others at $40 \%$ $(F(1,68)=7.92, p=0.006)$ and $60 \%(F,(1,68)=7.29, p=0.009)$ levels of intensity.

A dependent t-test identified both groups as being more accurate in identifying emotions on male faces $(M$ correct $=0.67)$ compared to female faces $(M$ correct $=0.59 ; t(-2.664), p=0.000)$. A $2 \times 2$ ANOVA revealed an interaction

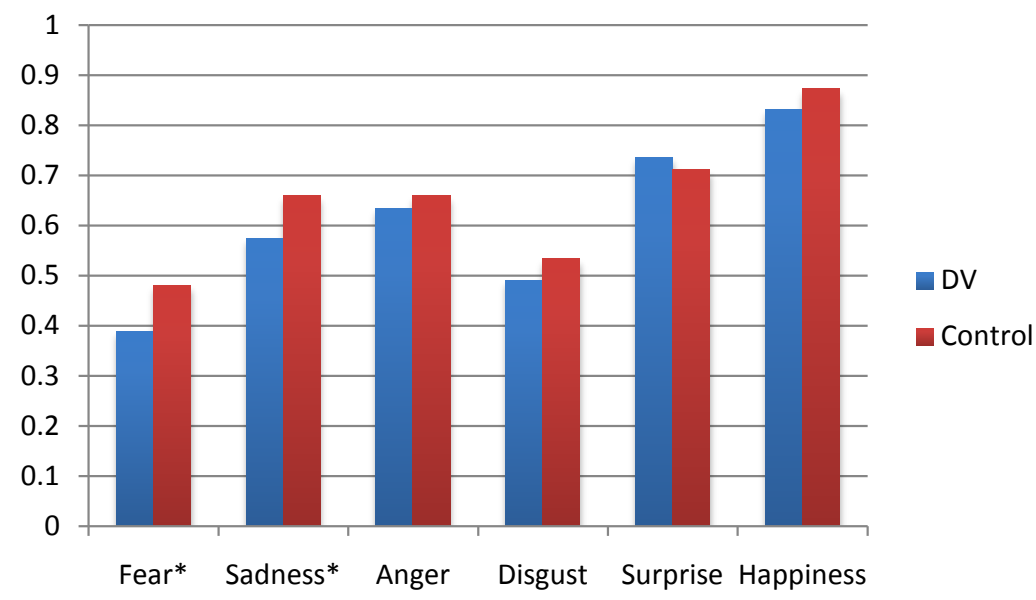

Note. ${ }^{\star}$ Indicates significant differences between DV and control groups.

Figure 1. Accuracy broken down by emotion. 
between gender of the faces being identified and whether the participant was in the DVO group or the control group $(F(1,68)=3.33, p=0.042)$. The results of the interaction suggest that when compared to the nonviolent control group, participants in the DVO group were less accurate in identifying emotions of female faces than male faces.

When demographic differences were controlled for, DVOs were still significantly less accurate at identifying the emotions of others after adjusting for age $(F(1,67)=8.47, p=0.037)$, race/ethnicity $(F(1,67)=7.45, p=0.008)$ and chemical dependency history $(F(1,67)=7.65, p=0.034)$. Significant differences in facial emotion recognition were no longer present when the ANCOVA method controlled for group differences in income $(F(1,65)=1.33, p=0.253)$ and education level $F(1,67)=1.79, p=0.185)$.

When mean GAMA scores were compared using a t-test, no significant difference between DVO participants and control participants $(t-3.226), p=$ $0.111)$ was found. DVO participants had mean scores in the average range ( $M=$ 103.86) while the control participants had mean scores in the high average range $(M=112.97)$. After controlling for GAMA scores using the ANCOVA covariate method, there were still significant differences between the DVO participants' and the control participants' overall ability to accurately identify the emotions of others $(F(1,68)=8.64, p=0.018)$.

\subsection{Self-Identified Empathy Measure}

When using a $2 \times 4$ ANOVA to analyze the different scales of the IRI, there were no significant differences between the domestic violence perpetrator participants and non-violent controls on the three scales of Fantasy $(F(1,68)=0.141, p=$ $0.708)$, perspective taking $(F(1,68)=0.037, p=0.848)$, and empathic concern $(F(1,68)=1.599, p=0.210$; Lauterbach \& Hosser, 2007) [15]. Nevertheless, a significant difference between the domestic violence participants and the control participants on the personal distress scale $(F(1,68)=5.228, p=0.025)$ emerged, with domestic violence perpetrator participants acknowledging a higher rate of personal distress in stressful situations $(M=10.26)$ compared to the control participants $(M=7.62)$.

A Pearson Product Correlation was used to analyze the correlation between each of the IRI scales and the participants' overall ability to accurately recognize facial emotions. In the domestic violence perpetrator group, there were no significant correlations between their facial emotion recognition accuracy and IRI scale scores with the exception of the relationship between the Perspective Taking scale and the Empathic Concern scale $(r=0.570, p<0.01)$. For the control participants, there were also several non-significant differences between facial emotion recognition accuracy and IRI scales apart from the relationship between the Empathic Concern scale and the Personal Distress scale $(r=0.452, p<0.01)$. Table 1 shows the correlations between each scale of the IRI and overall emotion recognition accuracy. 
Table 1. Interpersonal Reactivity Index (IRI) and Facial Emotion Identification for the DVO Group.

\begin{tabular}{cccccccc}
\hline & $\begin{array}{c}\text { Overall } \\
\text { Accuracy }\end{array}$ & $40.0 \%$ & $60.0 \%$ & $\begin{array}{c}\text { Perspective } \\
\text { Taking }\end{array}$ & $\begin{array}{c}\text { Fantasy } \\
\text { Scale }\end{array}$ & $\begin{array}{c}\text { Empathic } \\
\text { Concern }\end{array}$ & $\begin{array}{c}\text { Personal } \\
\text { Distress }\end{array}$ \\
\hline Overall Accuracy & 1 & 0.461 & $0.523^{* *}$ & -0.063 & 0.158 & -0.125 & 0.052 \\
$40.0 \%$ & $0.521^{* *}$ & 1 & 0.262 & -0.036 & 0.044 & -0.060 & 0.075 \\
$\begin{array}{c}\text { 60.0\% } \\
\text { Perspective }\end{array}$ & $0.534^{* *}$ & $0.520^{* *}$ & 1 & 0.003 & 0.263 & 0.022 & 0.114 \\
$\quad \begin{array}{c}\text { Taking } \\
\text { Fantasy Scale }\end{array}$ & 0.298 & 0.220 & 0.038 & 1 & 0.223 & $0.570^{* *}$ & -0.076 \\
$\quad \begin{array}{c}\text { Empathic } \\
\text { Concern }\end{array}$ & 0.018 & 0.028 & 0.14 & 0.193 & 1 & 0.266 & 0.104 \\
Personal Distress & -0.149 & 0.307 & 0.199 & -0.132 & -0.084 & 1 & 0.015 \\
\end{tabular}

Note: Domestic Violation (DV) correlations are in the upper right half of the table. Control correlations are in the lower left hand of the table. ${ }^{* *}$ Significant at the $p=0.01$ level.

\section{Discussion}

The present study sought to ascertain the relationship between self-identified empathy, demographic factors, and facial emotion recognition for DVO participants compared to a control group of nonviolent men. DVOs were significantly less accurate in identifying the emotions of fear and sadness compared to the control group. They were also less accurate in identifying emotions at $40.0 \%$ and $60.0 \%$ emotional intensity when all emotions (happiness, sadness, anger, disgust, fear, and surprise) were combined. Significant differences remained after controlling for race and ethnicity, age, and chemical dependence history. Significant differences in ability to accurately recognize the emotions of others were no longer present when annual income and highest level of education attained were accounted for, suggesting they may have a significant impact on emotion recognition accuracy. There was no significant difference in cognitive ability between the groups as measured by the GAMA and DVO participants remained less able to accurately identify the emotions of others after GAMA scores were accounted for.

No significant differences on the subscales of empathic concern, perspective taking, or fantasy were found. DVOs endorsed higher scores on the Perceived Distress subscale of the IRI. While both groups of participants were significantly less accurate in identifying the emotions of women compared to men, the DVO participants were significantly less accurate in recognizing the emotions of women compared to the control participants.

The findings of the present study add to the current literature of family violence etiology and therapy in a number of ways. The results contribute to the understanding of DVOs by identifying deficits experienced in accurately recognizing the emotions of fear and sadness. In addition, DVOs presented with deficits with more realistic emotional presentation at $40.0 \%$ and $60.0 \%$ intensity. Deficits in accuracy of emotion recognition for DVOs were not influenced by cognitive deficits as previously thought (Babcock et al., 2008) [13]. The high 
emotional reactivity DVOs reported experiencing in stressful situations influences their likelihood to react violently when misidentifying the emotional state of others, especially women. The current findings and previous literature suggest that DVOs may be even less accurate in identifying sadness and fear when in an escalating argument with their partners, leading to a higher likelihood of violence. A violent reaction based on an inaccurate identification of emotion as possibly anger or disgust rather than fear or sadness breaks down the emotional communication between partners and, ultimately, harms the relationship.

\subsection{Clinical Implications}

Presently, models of domestic violence offender therapy focus largely on cognitive awareness and fail to address how to accurately recognize a partner's emotions and effectively respond to the emotion once identified (Lehman \& Simmons, 2009) [24]. A more traditional, “...rational approach designed to confront established norms and beliefs about the perceived benefits of crime versus the perceived costs of crime" is the most commonly used approach (Walters et al., 2007 , p. 1425) [25]. In addition, current domestic violence treatment commonly focuses on identifying and disputing sexist views believed to be present with most DVOs. In the present study, the DVO group obtained a significantly higher score on the perceived distress scale of the IRI. A high perceived distress scale score indicates participants self-identified as having a higher level of emotional distress or reactivity in stressful situations. This reactive distress pattern is a key trait in individuals who have been identified as reactive/affectively violent offenders in the literature (Hanlon et al., 2013) [26]. A rational approach may be effective in proactive thinking but will likely have less impact on reactive thinking due to the impulsive and irrational nature of the aggression in the latter group.

Mental health professionals who work with DVOs can be more effective with reactive/affective type DVOs in a variety of ways. Awareness that male DVOs have a deficit in accurately identifying fear and sadness in female faces can be beneficial in increasing the effectiveness of DVO treatment. A deficit in accurately identifying the emotions of others makes it difficult for one to respond with emotional regulation in an appropriately empathic manner. An empathic connection with a potential victim motivates the offender to set in motion the mechanisms he has learned to keep from offending and eventually eradicate family violence (Grady \& Rose, 2011) [27].

A greater focus on active learning and enactment may be helpful to learn emotional information and emotion regulation application on a more visceral rather than cognitive level. Active learning could also involve informing DVOs of the emotion recognition deficits found in the literature to bring their possible limitations into awareness. Treatment would then involve participation in an applied computer program or role playing in which the DVO communicates verbally with his partner to inform her of the emotion he believes she is feeling 
and to invite her to verbally share her feelings (Sygel \& Kristiansson, 2014) [28]. This verbal communication may reduce ambiguity relying solely on emotion identification through facial expressions.

A reliance on merely teaching accurate emotion recognition to DVOs with the hope that they become as skilled as nonviolent controls is likely unrealistic. Given that child abuse and neglect is a common risk factor for future difficulty with accurate emotion identification, it is likely the DVOs' emotion recognition deficits stem from childhood and although it can be improved, it may not reach the baseline of the nonviolent control group (Ardizzi et al., 2013 [29]; Luke \& Banerjee, 2013 [4]). Communication training can help fill the gap that may be left by emotion-recognition training alone.

In addition, the strong own-gender bias in accurate facial emotion recognition held by the domestically violent men in the present sample may be the result of the need to strongly identify with one's own in-group. This may be the result of spending more time in the presence of one's own gender group compared to the opposite gender. Domestically violent men may have spent more time in the presence of other males during childhood and therefore, may be at a greater deficit in accurately identifying female faces compared to nonviolent controls. It could also be the result of the sexist views believed to be present in current domestic violence treatment models (Walters, Frederick \& Schlauch, 2007) [25]. If men who are perpetrators of family violence hold sexist attitudes toward women, they may work harder to, and therefore be better at, recognizing the emotions of men, whom they see as their equals, compared to women, whom they see as their inferiors. Therefore, common models of domestic violence therapy still need to be included. In addition to the common models, active learning should be integrated into current models of therapy especially in relation to facial emotions of fear and sadness.

\subsection{Methodological Implications}

The methodological strengths of this study build upon gaps in previous literature. For example, including a facial emotion recognition task with several different emotional intensities is a strength of the present study not addressed in previous literature. In addition, the present study addressed the hypotheses of previous studies that cognitive deficits may influence facial emotion recognition accuracy and in the present study, it was not found to have a significant impact. Continued use of a cognitive measure to account for possible cognitive deficits is recommended. Future studies would benefit from using a specific childhood trauma questionnaire to address trauma history, as well as, more specific measures used to differentiate between reactive/affective violent offenders and proactive/predatory violent offenders.

\subsection{Limitations and Future Directions}

Similar to the majority of existing literature, this study was limited by the con- 
venience sampling characteristics, thus influencing both internal and external validity. The control participant sample was significantly older, of a higher socioeconomic status, and more likely to be Caucasian than the domestic violence offender sample. Future studies would benefit from ensuring recruiting strategies to include a control sample matched to the domestic violence offender sample in the demographic factors of age, race, annual income, and highest level of education. In addition, due to the limited number of Nimstim faces to choose from, all faces in the facial emotion recognition task were Caucasian. This skews the data in that much like the own-gender bias, people demonstrate an own-racial bias as well and are better able to identify emotions in their own race (Scherf \& Scott, 2012) [30]. Another limitation of this study was the use of the LEC-5, which does not specifically ask about repeated traumas or childhood trauma. The LEC-5 likely did not tap into the increased exposure to violence in childhood many of the domestic violence offender participants likely experienced (Gardner et al., 2014 [3]; Murrell, Christoff \& Henning, 2007 [31]). A measure that focuses on childhood trauma specifically, and identifies repeated compared to one-time traumatic events, would ascertain what role childhood trauma may play in accuracy of emotion recognition.

Future directions would be to include lesbian, gay, bisexual, and transgender (LGBT) participants and female DVOs in future research of empathy and facial emotion recognition tasks. Given the previous research regarding own-gender bias and women's higher scores on empathy tasks and accuracy in facial recognition, coupled with fewer LGBT and women being mandated to participate in DVO treatment, the present study included only heterosexual men as participants. Due to these differences, theoretical and clinical implications of this study cannot be generalized to treatment with LGBT and women DVOs.

\section{Conclusion}

The present study aimed to understand the impact of self-identified empathy, cognitive ability, trauma history and demographic information on DVOs' ability to recognize facial emotions when compared to nonviolent men. Results suggest a significant difference between DVOs and nonviolent men, in which DVOs are significantly less accurate in identifying fear and sadness than nonviolent men. To add, DVOs also show greater impairment when recognizing emotions of female faces compared to male faces. The high emotional reactivity DVOs report experiencing in stressful situations may influence their likelihood to react violently when misidentifying the emotional state of others. These findings highlight the importance of including psychoeducation around facial emotion recognition in treatment for DVOs as a means to ameliorate family violence.

\section{References}

[1] Whitaker, D.J. and Lutzker, J.R. (Eds.) (2009) Preventing Partner Violence: Research and Evidence Based Intervention Strategies. American Psychological Association, Washington DC. https://doi.org/10.1037/11873-000 
[2] Jaffe, A.E., Simonet, D.V., Tett, R.P., Swopes, R.M. and Davis, J.L. (2015) Multidimensional Trait Emotional Intelligence and Aggressive Tendencies in Male Offenders of Domestic Violence. Journal of Family Violence, 30, 769-781.

https://doi.org/10.1007/s10896-015-9729-3

[3] Gardner, F.L., Moore, Z.E. and Dettore, M. (2014) The Relationship between Anger, Childhood Maltreatment, and Emotional Regulation Difficulties in Intimate Partner and Non-Intimate Partner Violent Offenders. Behavior Modification, 38, 779-800. https://doi.org/10.1177/0145445514539346

[4] Luke, N. and Banerjee, R. (2013) Differential Associations between Childhood Maltreatment Experiences and Social Understanding: A Meta-Analysis and Systematic Review. Developmental Review, 33, 1-28. https://doi.org/10.1016/j.dr.2012.10.001

[5] Barnett, G.D. and Mann, R.E. (2013) Cognition, Empathy, and Sexual Offending. Trauma, Violence, \& Abuse, 14, 22-33. https://doi.org/10.1177/1524838012467857

[6] Gery, I., Miljkovitch, R., Berthoz, S. and Soussignan, R. (2009) Empathy and Recognition of Facial Expressions of Emotions in Sex Offenders, Non-Sex Offenders and Normal Controls. Psychiatry Research, 165, 252-262. https://doi.org/10.1016/j.psychres.2007.11.006

[7] Covell, C.N., Huss, M.T. and Langhinrichsen-Rohling, J. (2007) Empathic Deficits among Male Batterers: A Multidimensional Approach. Journal of Family Violence, 22, 165-174. https://doi.org/10.1007/s10896-007-9066-2

[8] Book, A.S., Quinsey, V.L. and Langford, D. (2007) Psychopathy and the Perception of Affect and Vulnerability. Criminal Justice and Behavior, 34, 531-544. https://doi.org/10.1177/0093854806293554

[9] Brook, M. and Kosson, D. (2012) Impaired Cognitive Empathy in Criminal Psychopathy: Evidence from a Laboratory Measure of Empathic Accuracy. Journal of Abnormal Psychology, 22, 156-166.

[10] Marsh, A.A. and Blair, R.J.R. (2008) Deficits in Facial Affect Recognition among Antisocial Populations: A Meta-Analysis. Neuroscience Biobehavior Review, 32, 454-465. https://doi.org/10.1016/j.neubiorev.2007.08.003

[11] Bowen, E., Gilchrist, E. and Beech, A. (2005) An Examination of the Impact of Community-Based Rehabilitation on the Offending Behavior of Male Domestic Violence Offenders and the Characteristics Associated with Recidivism. Legal and Criminological Psychology, 10, 189-209. https://doi.org/10.1348/135532505X36778

[12] Domes, G., Hollerbach, P., Vohs, K., Mokros, A. and Habermeyer, E. (2013) Emotional Empathy and Psychopathy in Offenders: An Experimental Study. The Journal of Personality Disorders, 27, 67-84. https://doi.org/10.1521/pedi.2013.27.1.67

[13] Babcock, J.C., Green, C.E. and Webb, S.A. (2008) Decoding Deficits of Different Types of Batterers during Presentation of Facial Affect Slides. Journal of Family Violence, 23, 295-302. https://doi.org/10.1007/s10896-008-9151-1

[14] Hooper, L., Stockton, P., Krupnick, J. and Green, B. (2011) Development, Use, and Psychometric Properties of the Trauma History Questionnaire. Journal of Loss and Trauma, 16, 258-283. https://doi.org/10.1080/15325024.2011.572035

[15] Lauterbach, O. and Hosser, D. (2007) Assessing Empathy in Prisoners-A Shortened Version of the Interpersonal Reactivity Index. Swiss Journal of Psychology, 66, 91-101. https://doi.org/10.1024/1421-0185.66.2.91

[16] Gray, M.J., Litz, B.T., Hsu, J.L. and Lombardo, T.W. (2004) Psychometric Properties of the Life Events Checklist. Assessment, 11, 330-341.

https://doi.org/10.1177/1073191104269954 
[17] Davis, M.H. (1983) Measuring Individual Differences in Empathy: Evidence for a Multidimensional Approach. Journal of Personality and Social Psychology, 44, 113-126. https://doi.org/10.1037/0022-3514.44.1.113

[18] Naglieri, J.A. and Bardos, A.N. (1997) General Ability Measure for Adults. Pearson Assessments, Minneapolis, MN.

[19] Bardos, A.N. (2003) The General Ability Measure for Adults. In: McCallum, R.S., Ed., Handbook of Nonverbal Assessment, Kluwar Academic/Plenum Publishers, New York. https://doi.org/10.1007/978-1-4615-0153-4_8

[20] Tottenham, N., Tanaka, J.W., Leon, A.C., McCarry, T., Nurse, M., Hare, T.A., Marcus, D., Westerlund, A., Casey, B.J. and Nelson, C. (2009) The NimStim Set of Facial Expressions: Judgments from Untrained Research Participants. Psychiatry Research, 168, 242-249. https://doi.org/10.1016/j.psychres.2008.05.006

[21] Ekman, P. and Friesen, W.V. (1978) Facial Action Coding System: A Technique for the Measurement of Facial Movement. Consulting Psychologists Press, Palo Alto, CA.

[22] Pham, T.H. and Philippot, P. (2010) Decoding of Facial Expression of Emotion in Criminal Psychopaths. Journal of Personality Disorders, 24, 445-459. https://doi.org/10.1521/pedi.2010.24.4.445

[23] Peterson, E. and Peake Pohja, M. (2015) Child Maltreatment and Facial Emotion Recognition in a College Sample. Association for Psychological Science, New York.

[24] Lehman, P. and Simmons, C.A. (2009) Strengths-Based Batterer Intervention: A New Paradigm in Ending Family Violence. Springer Publishing Company, New York.

[25] Walters, G.D., Frederick, A.A. and Schlauch, C. (2007) Postdicting Arrests for Proactive and Reactive Aggression with the PICTS Proactive and Reactive Composite Scales. Journal of Interpersonal Violence, 22, 1415-1430. https://doi.org/10.1177/0886260507305556

[26] Hanlon, R.E., Brook, M., Stratton, J., Jensen, M. and Rubin, L.H. (2013) Neuropsychological and Intellectual Differences between Types of Murderers: Affective/Impulsive versus Predatory/Instrumental (Premeditated) Homicide. Criminal Justice and Behavior, 40, 933-948. https://doi.org/10.1177/0093854813479779

[27] Grady, M. and Rose, R. (2011) The Empathy Index: An Evaluation of the Psychometric Properties of a New Empathy Measure for Sex Offenders. Journal of Interpersonal Violence, 26, 3790-3814. https://doi.org/10.1177/0886260511403755

[28] Sygel, K. and Kristiansson, M. (2014) Reactions on Display/Intimate Partner Violence (RoD/IPV) - A Study of a New Interactive Computer Simulation Program for the Treatment of Men Convicted of Intimate Partner Violence. International Journal of Forensic Mental Health, 13, 369-380. https://doi.org/10.1080/14999013.2014.951104

[29] Ardizzi, M., Martini, F., Umilta, M.A., Sestito, M., Ravera, R. and Gallese, V. (2013) When Early Experiences Build a Wall to Others' Emotions: An Electrophysiological and Autonomic Study. PLoS ONE, 8, e61004. https://doi.org/10.1371/journal.pone.0061004

[30] Scherf, K.S. and Scott, L.S. (2012) Connecting Developmental Trajectories: Biases in Face Processing from Infancy to Adulthood. Developmental Psychobiology, 54, 643-663. https://doi.org/10.1002/dev.21013

[31] Murrell, A., Christoff, K. and Henning, K. (2007) Characteristics of Domestic Violence Offenders: Associations with Childhood Exposure to Violence. Journal of Family Violence, 22, 523-532. https://doi.org/10.1007/s10896-007-9100-4 